\title{
Editorial
}

\section{Toward the Next-Generation Peer-to-Peer Services}

\author{
Yi Cui, ${ }^{1}$ Ben Y. Zhao, ${ }^{2}$ and S. Chen ${ }^{3}$ \\ ${ }^{1}$ Department of Electrical Engineering and Computer Science, Vanderbilt University, VU Station B 351824, \\ Nashville, TN 37235-1826, USA \\ ${ }^{2}$ Department of Computer Science, University of California-Santa Barbara, 1123 Harold Frank Hall, \\ Santa Barbara, CA 93106-9560, USA \\ ${ }^{3}$ Department of Computer and Information Science and Engineering, University of Florida, CSE Building, \\ Room 460, Gainesville, FL 32607, USA
}

Received 11 December 2007; Accepted 11 December 2007

Copyright (C) 2007 Yi Cui et al. This is an open access article distributed under the Creative Commons Attribution License, which permits unrestricted use, distribution, and reproduction in any medium, provided the original work is properly cited.

Recent years have witnessed the blossom of the P2P application model on the Internet. Popular examples include P2P file sharing networks, streaming multimedia and content distribution networks, and P2P metaservices such as peerto-peer lookup services, reputation services, and network underlays. In this special issue, we aim to present both a timely retrospect of recent achievements in $\mathrm{P} 2 \mathrm{P}$ services and an outlook of new challenges ahead. The invited papers address a variety of topics, including the following.

The first paper, which is "A measurement study of the structured overlay network in P2P file-sharing systems," conducts a measurement study on the E-Mule overlay network. It presents a novel crawler program design that provides a perspective of overlay networks from a single user viewpoint, which helps identify new vulnerabilities such as DDOS attacks.

The second paper, "Analysis and implementation of gossip-based P2P streaming with distributed incentive mechanisms for peer cooperation," studies the randomized gossipbased scheme for P2P streaming and proves that an incentive mechanism can be created for a live streaming P2P protocol while preserving the asymptotic properties of the gossipbased scheme. It further proposes a functional architecture and protocol format for this new streaming paradigm.

The third paper, "Enhanced P2P services providing multimedia content," aims to remove the dependence of current P2P query systems on unique identifiers or keywords. It proposes an original image and video sharing system where users can interactively search for interesting resources using content-based image and video retrieval techniques.

The fourth paper, "A hybrid query scheme to speed up queries in unstructured peer-to-peer networks," studies the problem of locating resources in unstructured P2P networks.
By identifying problems in existing approaches such as flooding and random walk, the paper proposes a new hybrid query scheme that groups peers into clusters based on similar interests, and improves communication efficiency by mixing intercluster queries and intracluster queries.

The fifth paper, "Globally decoupled reputations for large distributed networks," points out the vulnerability of existing $\mathrm{P} 2 \mathrm{P}$ reputation systems to unfair rating attacks, and recommends that reputation systems decouple each peer's reputation as service provider from that as service recommender, making reputations more resistant to tampering. It further proposes a scalable approach to compute decoupled service and feedback reputations, and demonstrates the effectiveness of the new model against previous nondecoupled reputation approaches.

Yi Cui

Ben Y. Zhao

S. Chen 

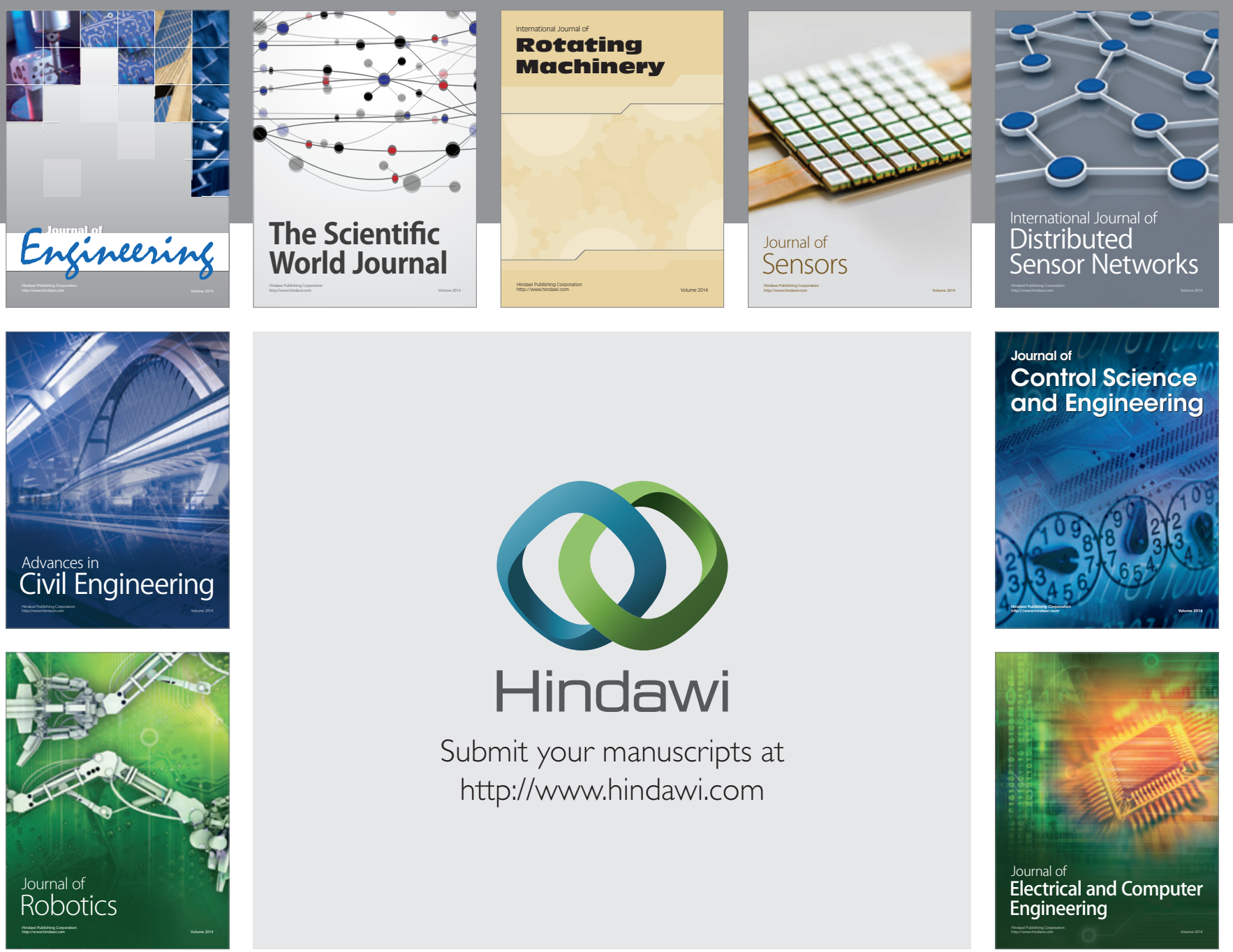

Submit your manuscripts at

http://www.hindawi.com
